\title{
AN INTELLIGENT MAXIMUM POWER POINT SOLAR TRACKING SYSTEM
}

\section{Nada Masood Mirza* \\ Al Ain University, United Arab Emirates}

This paper accentuates the enhancement of electric energy produced from Photo Voltaic (PV) solar modules as a result of sun tracking. The development of the tracking system is based on finding the orientation of a solar module at which maximum power is delivered. Two approaches for monitoring "Real-time power" have been demonstrated namely Pulse Width Modulation (PWM) based circuit and varying resistive load using analog switches. The approaches are meant to obtain Voltage-Current (VI) curve of the solar panel and consequently locate the Maximum Power Point (MPP). A modified form of the Perturb \& Observe (P\&O) algorithm has been used for tracking. The tracking system has many advantages in relation to similar devices. It is intelligent in a way that it not only performs real-time power monitoring but also has the ability to store information and improve behavior based on previous tracking history. The effectiveness of the proposed tracking scheme has been demonstrated experimentally on a commercially available single axis solar tracking platform.

Key words: MPP, PV, single axis tracker, solar tracking system

\section{INTRODUCTION}

One of the most important problems facing the world today is the energy crisis [1], [2]. To meet the growing demands of the world, with limited fossil fuels, research on methods of enhancing renewable energy sources are the need of the hour. The amazing thing about solar power is that it is easy to install and maintain. The major problem, however, lies in the low efficiencies of solar po wer systems. This inefficiency results in higher cost of solar solutions thus making solar solution mostly redundant in comparison to fossil fuel based energy resources.

The efficiency of a solar panel can be increased by a tracking system which can keep the panels oriented to get maximum power at the given instant.

A Maximum Power Point (MPP) tracking system can boost power output by $25-30 \%$. This increase in output of a solar panel because of tracking has been shown in Fig. 1

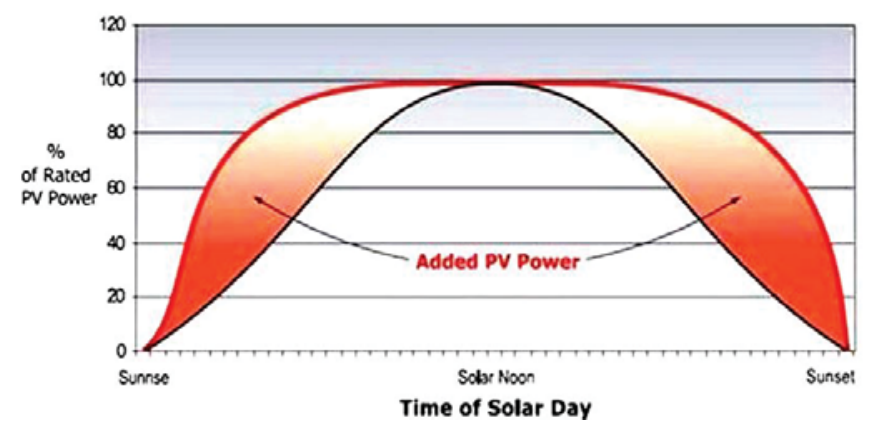

Figure 1: Increase in output upon solar tracking. The peak power time is increased because the solar cells are oriented in a way as to receive maximum solar irradiance
The MPP tracking system does not make use of conventional methods [4]- [6] of tracking the sun. Some systems use light sensors (photo diodes) which measure light but not the power. Some tracking systems have a pre-programmed database and thus require parameters like longitude, latitude, date and time. These types of trackers are unable to cater for cloud cover. In the case of MPP tracking, the MPP of a solar panel i.e., the point at which the solar panels deliver maximum power is the tracked variable and forms the basis of tracking the sun [4], [5].

Maximum power point is determined in real time for each angular position. The angle which gives the highest power output thus tells the tracker to orient itself accordingly. The V-I characteristic of a solar cell is non-linear and corresponds to the power as a function of the voltage given by the equation

$\mathrm{P}=\mathrm{V} \times \mathrm{I}$

$V_{o c}=\frac{k T}{q} \ln \left[\frac{I_{S C}}{I_{o}}\right]$

$I_{S C}=b G$

Where lo is the saturation current, $\mathrm{q}$ is the electronic charge, $\mathrm{k}$ is the Boltzmann constant, $\mathrm{T}$ is the absolute temperature, $\mathrm{G}$ is incident light intensity and $\mathrm{b}$ is a constant, depends on the properties of the semi-conductor junction, the geometry of the detector and the size of the collector area. To get the maximum power from a solar array, the solar cells must always be operated at or very near the point where the power curve is at a maximum, its peak point as shown in Fig 2.

However, this operating point keeps changing constantly due to ambient conditions which include solar irradiance, temperature, moisture in the air and intensity of 
light. These factors alter the V-I characteristic shifting the operating point where maximum power is delivered. As a result, we need to constantly track the power curve and keep the solar tracker oriented in a position where the maximum amount of power can be drawn [7]. The solar irradiance is a characteristic that deals with the amount of sun energy reaching the ground. The irradiance reaching the earth in ideal conditions is $1000 \mathrm{~W} / \mathrm{m}^{2}$. However, this value is altered significantly depending on geographical location, the angle of the sun, and the amount of haze or cloud cover preventing all of the sun's energy from reaching the ground. Output of solar cells is strictly affected by the changing irradiance. [8] - [10].

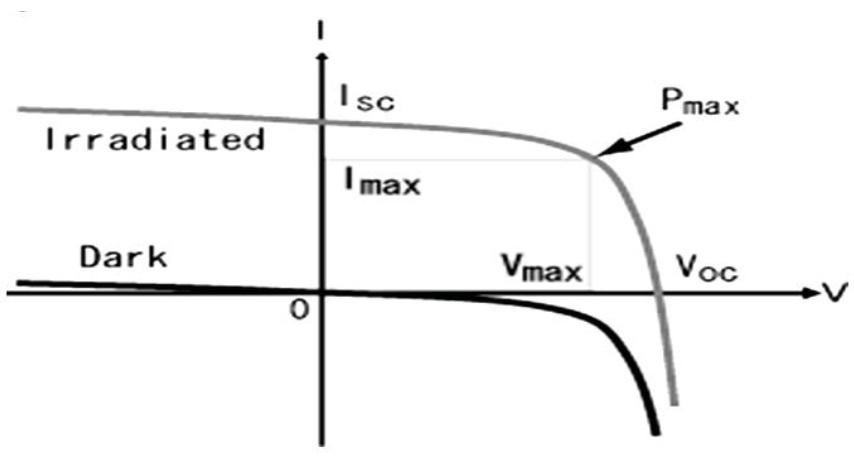

Figure 2: Solar V-I Characteristic curve: The knee of the curve represents the point where $V \times I$ maximize and maximum power is delivered

\section{BASIC DESIGN}

The fundamental approach involved the designing and subsequent manufacturing of a solar tracker featuring single axis operation. The tracker was visualized to be self (solar) powered and be able to learn from previous data, calculate MPP quickly, with very low power consumption and track angle with an accuracy of around 0.5 degrees.

The methodology adopted was to use a small 10-watt solar panel on a structure capable of supporting up to eight 250 watt panels. This 10 -watt panel will not only be used to power our circuitry and charge the battery but also be used to constantly monitor the MPP of the V-I curve of the panel. This MPP would be used by our algorithm to perform subsequent tracking.

Furthermore, it was desired that the tracker be independent of the geographical location or the time of operation. In order to achieve this plug and play characteristic the algorithm was developed to be able to accumulate the data initially and analyzing it to adapt itself according to the time and installation location of the solar tracker. The design also kept a balance between the power obtained through tracking and the power consumed in tracking. The solar tracker instead of tracking for a particular angle or following a photo sensor output calculates the power of the solar panel in real time [11] and orients the panels in such a way as to track for the maximum possible power.

\section{MECHANICAL DESIGN}

Pro-Engineer software has been used for 3D solid modeling and analysis of the tracking platform. The design complies with the dimensions of our practical model and material of their respective parts as shown in Fig 3. The stress strain analysis and dynamic analysis of the new model has been performed.

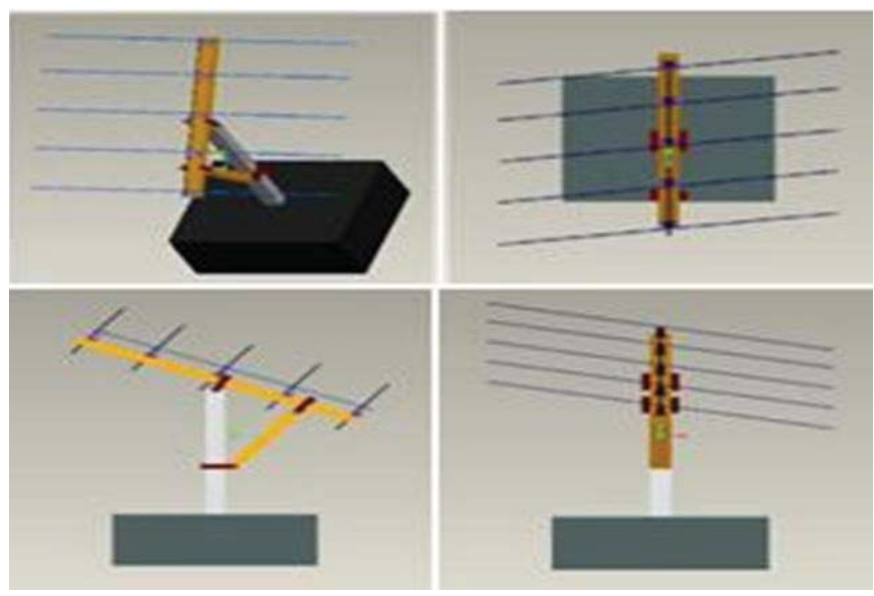

Figure 3: 3D Top, Side and Front view

\section{A. Hardware Simulation}

Basic function of our mechanical design depends upon the linear movement of an actuator. The model of joint actuator has been designed in Matlab Simulink and has been given proper inputs to get the required linear outputs. The joint actuator has been selected because it can be given linear signals so as to replicate our actual tracking platform.

\section{B. Drive Mechanism and Angle Measurement:}

A linear actuator with a ball screw mechanism is used for moving the tracker which supports up to $300 \mathrm{~kg}$ load and uses about 2 Amperes of current. An integrated motor driver (LMD18200) has been used to drive the actuator. It has the capability to provide sufficient current and also provides indicators such as motor current and thermal flag to ensure safe operation as the tracker moves 180 degrees from east-to -west.

The linear actuator is also equipped with a reed sensor, which gives 1305 pulses across east-west operation.

The angular resolution is given by the formula:

minimum angle $=\frac{90^{\circ}}{1305}=0.0689^{\circ} \cong 0.07^{\circ}$

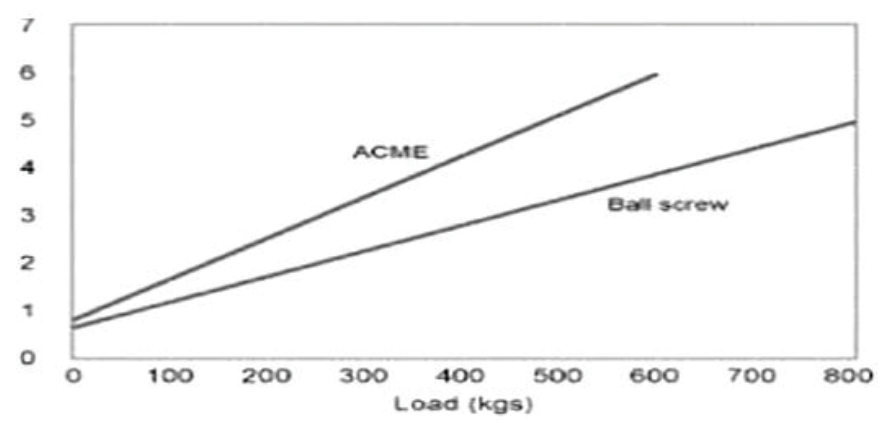

Figure 4: Load vs. Current behavior of linear actuator 


\section{ELECTRONIC DESIGN}

The electronics design consists of three basic portions namely panel MPP calculation circuit, a microcontroller interface and signal conditioning for reed sensor.

\section{Maximum Power Point Calculation}

A solar cell may operate over a wide range of voltages (V) and currents (I) as shown in Fig 2. By increasing the resistive load on an irradiated cell continuously from zero (a short circuit) to a very high value (an open circuit) one can determine the maximum-power point, the point that maximizes $\mathrm{V} \times \mathrm{l}$; that is, the load for which the cell can deliver maximum electrical power at that level of irradiation as shown in Fig 2. Maximum power is typically produced with $75 \%-80 \%$ of $\mathrm{VOC}$ and $90 \%$ of ISC. The short-circuit current (ISC) from a cell is nearly proportional to the illumination, while the open-circuit voltage (VOC) may drop only $10 \%$ with an $80 \%$ drop in illumination, so measuring the voltage does not provide accurate data for tracking [12].

Two separate methods have been employed for calculation of MPP, one involves PWM switching to control the flow of current across a resistor [11]. The second technique involves using analog switches in a binary sequence and varying current in $2 \mathrm{n}$ steps, where

' $n$ ' is the number of analog switches. In our design we have used 06 switches which give 64 unique values of current and voltage. This is essentially 64 different points on the IV curve of the solar panel. The maximum power point is determined via interpolation techniques on the accumulated data.

\section{PWM based MPP Calculation}

This technique is based upon high speed switching using Pulse Width Modulation (PWM) to control the current flow in the circuit. From the given panel (10W), closing the switch for very short period of time and taking the reading is used essentially make a virtual resistor as shown in Fig 6. A resistor by definition limits the flow of electrons in a circuit; this design limits flow of electrons by varying the PWM duty cycle [13], [14]. The value of the resistor is based upon the factors dictated by the panel. For a VOC of 19.2 volts and ISC of $700 \mathrm{~mA}, 10$ Watt solar panel, the min Resistor value is calculated by the formula:

$R_{\min }=\frac{0.85 \times V_{o c}}{0.9 \times I_{s c}}$

\section{MPP Calculation by Varying Resistive Loads}

The second MPP calculation is based on using analog switches to vary the resistive load on the panel and determine different points on the V-I curve and calculate the MPP. The number of points that can be determined is related to number of analog switches. A total of $2 n$ values are obtained from $n$ switches. Switches are turned $\mathrm{ON}$ in binary sequence with the resistors varying from highest

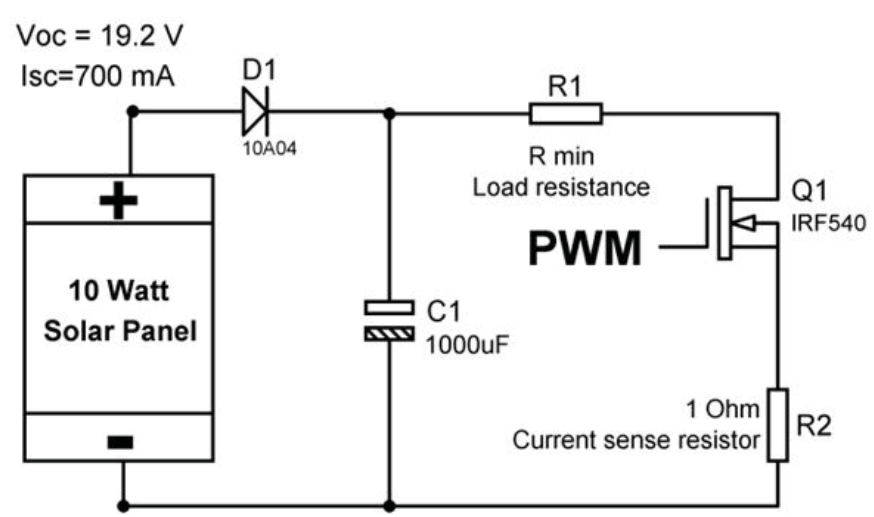

Figure 5: PWM based MPP calculation circuit

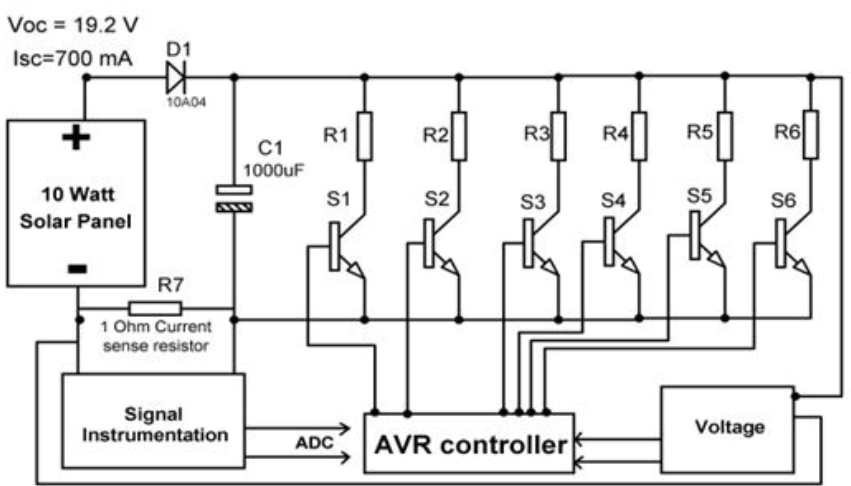

Figure 6: Analog switch based MPP calculation circuit

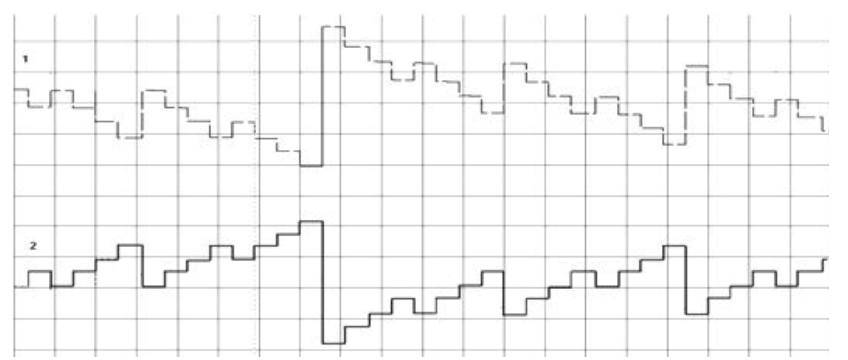

Figure 7: This is the DSO output of the analog switching MPP circuit. The upper curve represents the drop in voltage of the solar panel as a result of the load applied to it via the resistors while the lower curve indicates the rise in current

to lowest. The resistor values of the switches are 330, 120, 100, 82, 100and 270 Ohms respectively.

Each switch is turned ON for about $7 \mathrm{~ms}$, and voltage and current readings are taken, the signals are conditioned via an instrumentation amplifier, which is specifically designed for battery powered data acquisition systems.

Owing to the fast response of this approach in comparison to the PWM technique, this varying resistive load technique was employed. Each voltage and current value gives a point of the IV curve, Fig 7 shows the operation of the circuit and in this manner the Maximum power point is calculated with very low power loss.

\section{Microcontroller}

The tracking algorithm has been implemented on the avr atmega32 controller. The controller is relatively inexpen 
sive and has multiple built in features. Owing to the analog to digital conversion (ADC) channels of atmega32, the circuitry has been greatly reduced. ADC channels on-board the microcontroller have been used for the following purposes:

1. Reed switch pulse monitoring

2. Solar Panel current

3. Solar Panel voltage

4. Battery voltage level

5. Actuator current consumption

An entire port has been dedicated for the MPP circuitry through which a binary combination is given to the corresponding circuit and at the same time the current and voltage is measured. These values of voltage and current are used to measure power at varying resistive loads and thus locate the MPP of the solar panel.

The controller is also used for controlling the motor driver IC and charging the battery. Furthermore, the controller protects the battery from over charging. The ADC channels have also been used for feedback from the actuator regarding the current position of the panel.

\section{Reed Sensor}

The reed switch pulses contained a bit of noise but this was removed by simply monitoring the analog value. A pulse is considered if the value remains above 4.5 volts for more than 200 milliseconds and remains less than 0.25 volts for more than 70 milliseconds. This time of 200 ms and 70 ms was observed practically with the help of an oscilloscope as shown in Fig.8.

Reed sensor output has been carefully monitored because every pulse is of immense importance and miscalculation of one pulse can result in a huge discrepancy in the actual angle of the solar tracker and the processed angle. Based on the importance of this input of controller, ADC channel has been used in noise reduction mode. This implies that an accuracy of less than 0.1 degrees can be achieved as evident from equation (4).

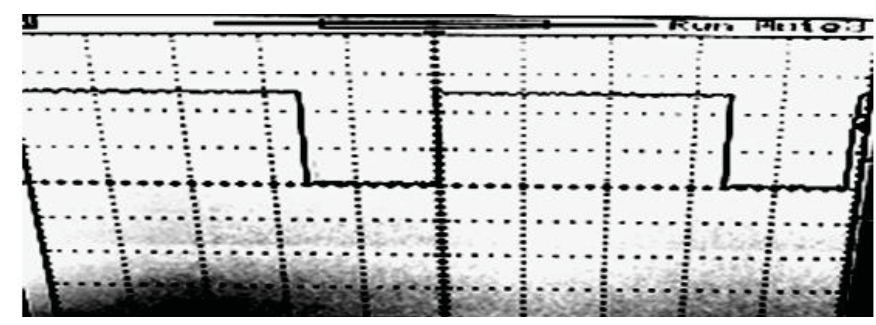

Figure 8: Output waveform of actuator reed sensor

\section{TRACKING STRATEGY}

Tracking the sun to a greater accuracy in an efficient manner is the core for MPP tracking strategies. The most widely used technique is the real-time realization of the gradient following method termed as Perturb and Observe $(P \& O)$ [15]. The $P \& O$ is simple and efficient since it does not require modeling the source characteristics and thus is independent of ageing, shadowing and other irregularities [16].

\section{Basics of Perturb \& Observe}

The P\&O involves observation of power $P(n)$ at the angle ' $n$ ' followed by a perturbation in an arbitrary direction. Now again the power $P(n+1)$ is measured. The difference in the power $\Delta \mathrm{P}$ observed at the two angles is the deciding factor for this algorithm. If " $\Delta P$ " from (6) is negative, the direction of perturbation will be reversed. Otherwise if " $\Delta \mathrm{P}$ " is positive; the panels will be moved in the same direction until the $\Delta \mathrm{P}$ comes out to be negative [14] as shown in Fig. 9

$$
\Delta P=P(n+1)-P(n)
$$

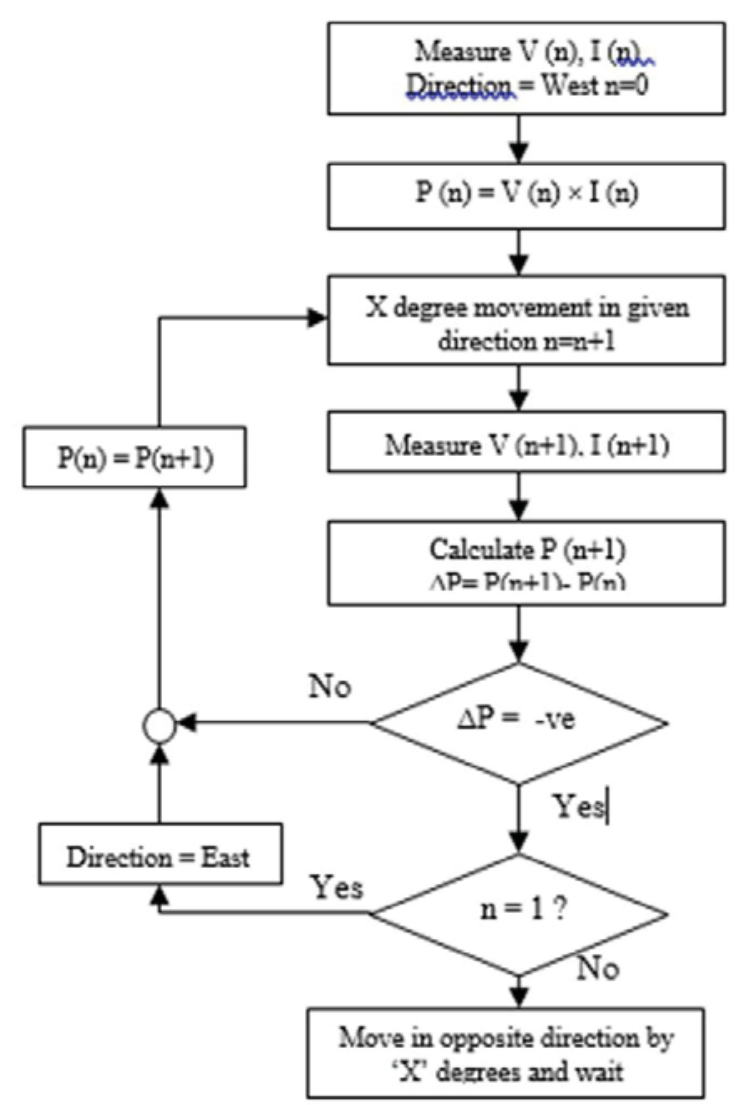

Figure 9: Flow chart of P\&O operation

\section{Implementation of $P \& O$}

$\mathrm{P} \& O$ is concerned only with finding the MPP angle for the instant under consideration. Our vision is tracking the sun continuously. For this purpose, our system follows the mentioned procedure. Initially, the system adjusts the day night cycle according to the flowchart in Fig 9. During this crucial first day the system keeps on tracking using the $\mathrm{P} \& \mathrm{O}$ with a waiting time of five minutes and scanning step " $X$ " of 0.7 degrees. In a similar manner on the second and third day the system again tracks the sun with the same parameters of scanning step. 


\section{Fine tuning of $P \& O$}

Once the data of first three relatively sunny days has been accumulated, P\&O can be modified in order to minimize the number of perturbations required for detecting the optimum angle. This can be achieved simply by comparing the data of post morning first angle observed by the system in the past three days. The difference in the angle between day 1 and day 2 i.e., $\Delta 1$ and similarly the difference between the angle on day 2 and day 3 i.e., $\Delta 2$ is averaged out to $\Delta a v$.

$\theta_{n}=$ First Angle on day ' $n$ '

$$
\begin{aligned}
& \Delta 1=\theta_{1}-\theta_{0} \\
& \Delta 2=\theta_{2}-\theta_{1} \\
& \Delta a v=\frac{\Delta_{1}+\Delta_{2}}{2} \\
& \theta_{\text {expected }}=\theta_{2}+\Delta_{a v}
\end{aligned}
$$

This $\theta$ expected is instrumental in saving the time and power consumption of the tracker. Since the tracker has been synchronized with the sunrise and sunset time, so this expected angle is very close to the actual angle on fourth day. Owing to this prediction the scanning step of $\mathrm{P} \& \mathrm{O}$ is reduced from 0.7 degrees to 0.14 degrees. This minute scanning takes place by the panel directly moving to the expected angle instead of scanning after every 0.7 degrees. This increase in accurate prediction is evident from Table 1. On the completion of day four of operation, the memory is updated such that the data of day 2 is saved as day 1 and so on so forth. This results in the tracker retaining only the data of previous three days and consequently saving precious memory of the microcontroller.

\section{Seasonal Changes and Cloud Cover Compensation}

Moreover, since the tracker is calibrated with sunrise and sunset timings so it automatically adjusts the changes in day night cycle due to the change in tilt of earth annually. Cloud cover is a very important factor to be considered while tracking. This factor is compensated by the data of power received at every angle saved in the memory. Each time the tracker reaches the expected angle, before fine tuning the angle, the power is observed and if

Table 1: Data for 73"08" east 33"40" north for 2009

\begin{tabular}{|c|c|c|c|}
\hline June & $\begin{array}{c}\text { Azimuth } \\
\text { Angle }\end{array}$ & March & $\begin{array}{c}\text { Azimuth } \\
\text { Angle }\end{array}$ \\
\hline 24th & 61.1 & 5th & 97.0 \\
\hline 25th & 61.1 & 6th & 96.5 \\
\hline 26th & 61.2 & 7th & 96 \\
\hline Actual(27th) & 61.2 & Actual (8th) & 95.5 \\
\hline Expected & 61.25 & Expected & 95.5 \\
\hline Difference & 00.05 & Difference & 00.00 \\
\hline
\end{tabular}

this power is less than $10 \%$ of previous day's power, this implies cloud cover. In this scenario the data for today for the current step is overwritten by yesterday's data and meanwhile the tracker performs a P\&O with a scanning step of 0.7 degrees as opposed to 0.14 degrees. Once 5 minutes have been passed, the tracker is moved to the next expected angle and the same process is repeated.

\section{RESULTS AND OBSERVATIONS}

This tracker was instrumental in proving the fact that tracking indeed increases the power output and especially during summer months when a tracker can result in up to $50 \%$ increase in output of solar modules. A comparison between Chronological, stationary and active power based sensing on a cloudy day. The figure shows that active power based sensing is the best option. On a cloudy day the results between

chronological and power based would be less as compared to a cloudy day. This is because of the inability for chronological tracking to compensate for clouds.

Despite the fact that chronological gives an increase in power as compared to stationary panels, the MPP tracker gives a relatively larger increase in output. Moreover, the chronological is unable to compensate the cloudy weather situation as efficiently a MPP tracker which continuously tracks the power of the solar panel. Furthermore it was observed that the voltage showed little changes during environmental changes whereas the current was highly sensitive. Throughout the day (sunny) voltage remained relatively stable. It is the current of the solar module that exhibited greatest fluctuations and thus dictated the MPP of the solar panel as shown in Fig. 11.

Moreover, it was observed that the simple prediction technique was very effective in predicting the position of the sun. The difference between the predicted and the actual angle for MPP quickly diminished to negligible amounts in a few day's operation as shown in Fig. 12

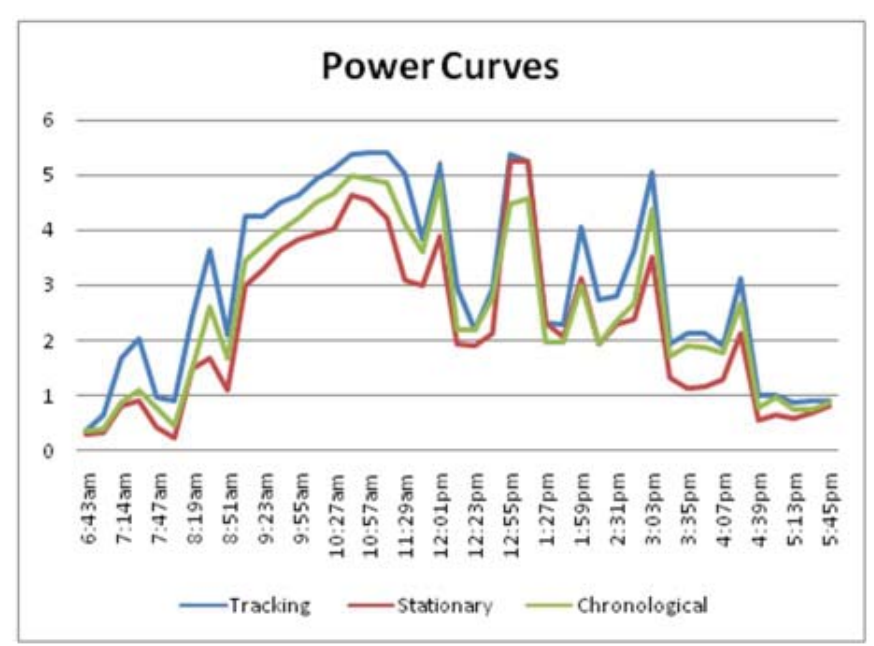

Figure 10: Power curve comparison 

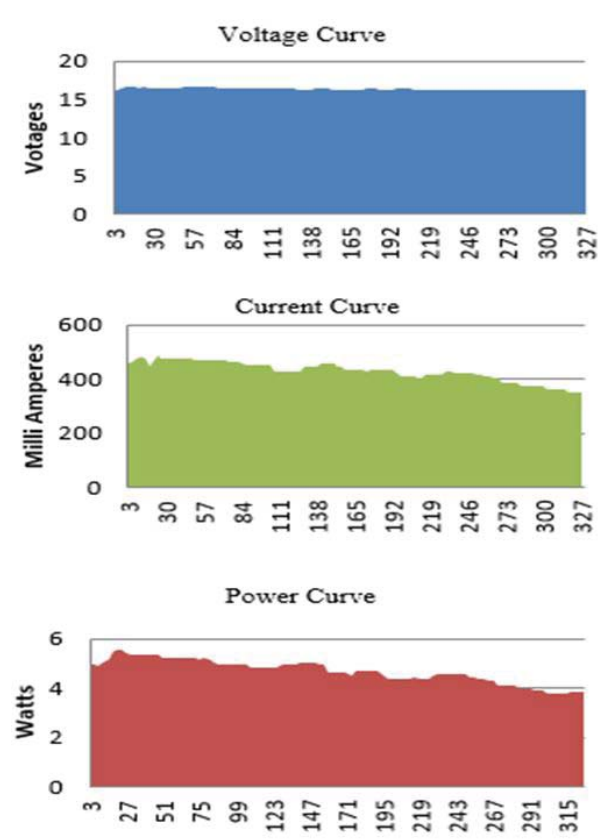

Figure 11: Voltage, Current and Power curves from 10 am till 3:30 pm showing the dependency of power on current and relative stability of voltage

\section{CONCLUSION}

The research carried out effectively proves the efficiency of a MPP tracking system not only as compared to stationary panels but also as in comparison with other tracking techniques like sensor based and chronological trackers. Moreover, it is evident that voltage or current alone cannot be secure parameters for tracking rather it is the MPP of the V-I curve of the panel which can be employed as a reliable tracking parameter. The prediction technique, simple and logical, owes its success to the synchronization of the tracker with the sunrise and sunset timings. More work needs to be carried out for finding the MPP of the panel in a fast, precise and simple manner.

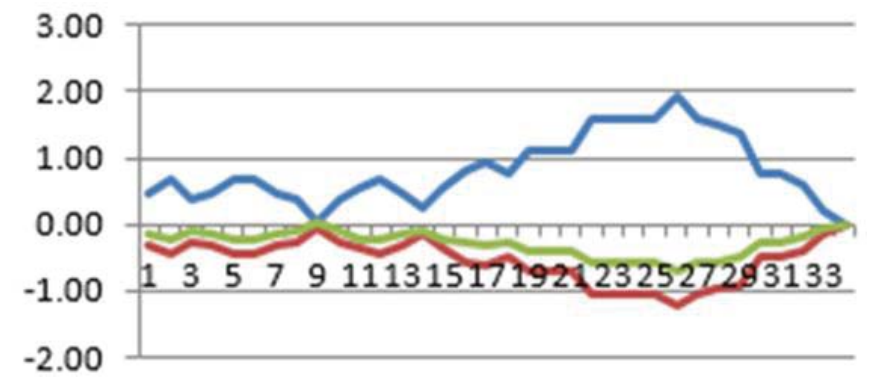

Figure 12: Horizontal axis represents the actual orientation where as blue, red and green curves represent the predicted angles of 26th,27th and 28th June 2009 (respectively) the panel at the tracker's testing location

\section{REFERENCES}

1. Khalil, A.A., \& El, S.M. Position control of Sun tracking system. page 45, Volume 6.

2. Energy crisis for the Earth.www.paulchefurka.ca/energygap.html.

3. Statistical review of energy of 2008. www.bp.com.

4. Saigh, A.A.M., \& ed., (1977). Solar Energy Engineering. New York: Academics press.

5. Nevile, R.C. (1995). Solar Energy Conversion in the Netherlands. Elsevier Science.

6. Clifford, M.J., \& Eastwood, D. Design of a Novell Passive Solar Tracker.

7. Anderson, E., \& Dohan, C. Solar Panel Peak Power Tracking System.

8. Klaus, B., \& Markvert, (1994). Solar Electricity Christeler. NY: Wiley Press.

9. National Center of Photovoltaic. Turning Sunlight into Electricity. www.eren.doe.gov/pv/siatomic.html.

10. Rubio, F.R., Ortega, M.G., Gordillo, F., \& LópezMartínez, M. (2007). Application of new control strategy for sun tracking. Energy Conversion and Management, 48(7), 2174-2184. doi:10.1016/j.enconman.2006.12.020

11. Figueiredo, J.M.G., \& Sá, d.J.M.G. Intelligent Sun-Tracking System for Efficiency Maximization of Photovoltaic Energy Production.

12. Grandi, G., Rossi, C., \& Casadei, D. A MPPT Algorithm For Single-Phase Single-Stage Photovoltaic Converters.

13. Rubio, F.R., Ortega, M.G., Gordillo, F., \& Lo'pezMartı'nez, M. Application of new control strategy for sun tracking.

14. Faranda, R., \& Leva, S. Energy comparison of MPPT techniques for PV Systems.

15. Pandey, A., Dasgupta, N., \& Mukerjee, A.K. High-Performance Algorithms for Drift Avoidance and Fast Tracking in Solar MPPT System.

16. Yafaoui, A., Wu, B., \& Cheung, R. Implementation of Maximum Power Point tracking algorithm for residential photovoltaic systems.

Paper submitted: 06.02.2019.

Paper accepted: 11.09.2019.

This is an open access article distributed under the CC BY-NC-ND 4.0 terms and conditions. 